applies to future use of tissue collected for a specific research project. Public opinion surveys in the United States reveal that some people do not want their tissues used for certain kinds of research ${ }^{2}$. Even if residual tissue is coded or anonymized, the potential exists for social, psychological and other harms to accrue to individuals, families and identifiable populations because the tissue and associated data may be linked to geographic region or ancestry (for example, tissue and data from Ashkenazi Jews, named Native American tribes or other indigenous peoples) ${ }^{2}$. Additional concerns have been raised about anonymizing residual tissue without obtaining consent to do so, and about anonymizing without consent tissue collected for specific research purposes that investigators later want to use in different studies $^{2,3}$. Although it is true that the US Office for Human Research Protections (OHRP) issued a guidance indicating that research with tissue and data that is coded and for which the investigator cannot 'readily 을 ascertain' identifiable private information about the source of tissue or information does not constitute human subjects research, this is not a legally binding regulatory requirement. Indeed, institutional review boards (IRBs) do not necessarily follow this guidance, as some may require IRB review and approval of studies using coded and/or anonymous samples.

Because millions of stored residual tissues were collected years ago without consent for research purposes (and many were obtained for research but without consent for secondary uses), an argument can be made that an acceptable alternative to the cost and effort of contacting individuals for consent for research use of these stored samples is ethics board approval for anonymizing tissues. However, it is difficult to justify continuing the practice of collecting tissue in treatment and diagnostic settings, and specifically for research purposes, without obtaining consent for future research use ${ }^{2}$. That such practices took place in the past does not mean that they should continue.

Because my article addressed the ethical and policies issues involving genetic research in the context of describing and comparing population biobank projects (Table 1 in my original article), many of the nuances that van Veen points out were unavoidably lost. However, van Veen's comments reinforce the theme of the piece: that there is a patchwork of ethical and regulatory policies regarding genetic research with human tissues. van Veen's comments also reveal that there remains disagreement over what constitutes ethical research with human tissues and that what is legal and customary may not be the same as what is ethical.

1. National Conference of State Legislatures. State Genetic Privacy Laws (http://www.ncsl.org/programs/ health/genetics/prt.htm).

2. Weir, R.F. \& Olick, R.S. The Stored Tissue Issue (Oxford Univ. Press, Oxford, UK, 2004).

3. Clayton, E.L. J. Law Med. Ethics 23, 375-377 (1995).

\title{
Transgenic plant science priorities
}

\section{To the editor:}

A letter by Vain in the November issue (Nat. Biotechnol. 23, 1348-1349, 2005) reports a study of two major science literature databases and tracks broad trends in plant transgenic science knowledge from 1973 to 2003. The study identified 30,624 papers, of which $14.8 \%$ related to technology development, $71.3 \%$ related to applications of technology and $13.8 \%$ related to development of genetically modified (GM) crops or feed. The author expresses the concern that the expanding gap between technology applications and development since the 1980s may limit future transgenic science and the ability to address issues related to GM crops.

One of the issues related to GM crops is

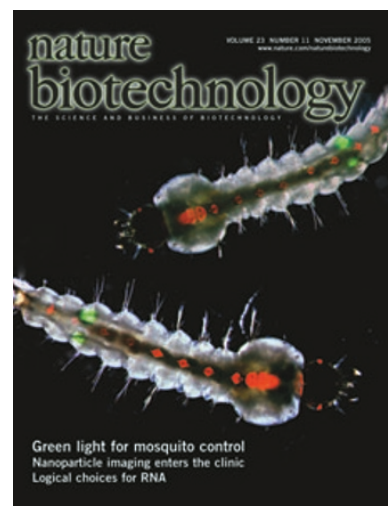
food safety. Vain's study did not seek to quantify the literature on this topic, but some studies using a more limited database have done so. One study's author searched the US National Library of Medicine Medline publications and documented 101 papers containing the terms 'food safety' and 'genetically engineered foods. ${ }^{1}$ Only eight of these papers reported findings from original rodent studies, with most of the remaining papers offering opinions and commentaries without supporting data. A search of the US Department of Agriculture's Current Research Information database (http:// cris.csrees.usda.gov/) from 1994 to 2002 identified 3,041 funded research projects related to plants and to biotech and/or transgenics, of which 145 related to toxins and 19 related to allergens ${ }^{2}$. Examination of these abstracts revealed that most of the toxin studies focused on enhancing pest protection through the use of plant toxins. Two of the toxin studies and five of the allergen studies were specifically using transgenic methods to study or alter known human toxins, allergens or allergenic foods. Two projects in 2001 sought to develop an animal model to test for unexpected allergens in GM foods. None of the 3,041 transgenic plant projects related to the appearance of unintended toxins or allergens in GM foods. It would be of interest to use Vain's more comprehensive databases to further characterize the empirical science on the safety of GM foods.

The paucity of studies on food safety has hampered national and international efforts to develop regulations and has been noted by two committees of the US National Academy of Sciences, both of which have recommended expanded research in this area $^{3,4}$. Similarly, a paucity of experimental studies related to ecological risks has been documented ${ }^{5}$ and led an Academy committee to identify a number of highpriority research topics to better inform the development of regulations ${ }^{6}$. More broadly, these findings both document that transgenic research has overwhelmingly emphasized technology application over basic or risk-related research and suggest a need to examine transgenic funding priorities.

\section{David Pelletier}

Cornell University, Division of Nutritional Sciences, 378 MVR Hall, Ithaca, New York 14853, USA

e-mail:dlp5@cornell.edu

1. Domingo, J. Science 288, 1748-1749 (2000).

2. Pelletier, D. Nutrition Reviews 63, 210-223 (2005)

3. National Research Council.Genetically Modified PestProtected Plants: Science and Regulation (National Academies Press, Washington, DC, 2000).

4. Institute of Medicine. Safety of Genetically Engineered Foods: Approaches to Assessing Unintended Health Effects (National Academies Press, Washington, DC 2004).

5. Wolfenbarger, L. \& Phifer, P. Science 290, 20882093 (2000).

6. National Research Council. Environmental Effects of Transgenic Plants: The Scope and Adequacy of Regulation (National Academies Press, Washington, DC, 2002). 